Pacific Journal of Mathematics

AN EXAMPLE OF A SIMPLE TRIOD WITH SUBJECTIVE SPAN 


\section{AN EXAMPLE OF A SIMPLE TRIOD WITH SURJECTIVE SPAN SMALLER THAN SPAN}

\section{A. LELEK}

The span of a metric space is the least upper bound of numbers $\alpha$ such that, roughly speaking, two points can move over the same portion of the space keeping a distance at least $\alpha$ from each other. The surjective span is obtained if it is required that, in addition, the whole space be covered by each of the moving points. These geometric ideas turn out to be important in continua theory. In the present paper, a simple triod is constructed such that the span of it is strictly greater than the surjective span.

Let $X$ be a connected metric nonempty space. By $p_{1}$ and $p_{2}$ we denote the standard projections of the product $X \times X$ onto $X$, that is, $p_{1}\left(x, x^{\prime}\right)=x$ and $p_{2}\left(x, x^{\prime}\right)=x^{\prime}$ for $\left(x, x^{\prime}\right) \in X \times X$. The surjective span $\sigma^{*}(X)$ [resp., the surjective semispan $\sigma_{0}^{*}(X)$ ] of $X$ is defined to be the least upper bound of the set of real numbers $\alpha$ with the following property: there exist connected sets $C_{\alpha} \subset X \times X$ such that $\alpha \leqq \operatorname{dist}\left(x, x^{\prime}\right)$ for $\left(x, x^{\prime}\right) \in C_{\alpha}$ and $p_{1}\left(C_{\alpha}\right)=p_{2}\left(C_{\alpha}\right)=X$ [resp., $\left.p_{1}\left(C_{\alpha}\right)=X\right]$. The span $\sigma(X)$ and the semispan $\sigma_{0}(X)$ of $X$ are defined by the formulae:

$$
\begin{aligned}
\sigma(X) & =\operatorname{Sup}\left\{\sigma^{*}(A): \varnothing \neq A \subset X, A \text { connected }\right\} \\
\sigma_{0}(X) & =\operatorname{Sup}\left\{\sigma_{0}^{*}(A): \varnothing \neq A \subset X, A \text { connected }\right\} .
\end{aligned}
$$

It follows directly from the definitions that the following inequalities hold:

$$
\begin{aligned}
& 0 \leqq \sigma^{*}(X) \leqq \sigma(X) \leqq \sigma_{0}(X) \leqq \operatorname{diam} X \\
& 0 \leqq \sigma^{*}(X) \leqq \sigma_{0}^{*}(X) \leqq \sigma_{0}(X) \leqq \operatorname{diam} X \\
& \sigma(A) \leqq \sigma(X), \quad \sigma_{0}(A) \leqq \sigma_{0}(X) \quad(A \subset X)
\end{aligned}
$$

It is not difficult to check that the above definition of the span, formula (1), is equivalent to the definition given in [5]. Continua of surjective span zero were defined in [10]. For each arc, as well as for each arc-like continuum, all these four quantities are equal to zero (cf. [8], Propositions 1.3 and 2.1). Nevertheless, they are quite useful in the theory of tree-like continua (see [2], [3], [6], [7] and [8]). From this 
view-point, the relationship between different kinds of span of a tree-like continuum seems to be of some importance. There is an easy example of a continuum $X$ such that $\sigma(X)=\sigma_{0}(X)=1$ and $\sigma^{*}(X)=\sigma_{0}^{*}(X)=\frac{1}{2}$ (see [8], Example 1.4). Its existence shows, among other things, that the analogues of inequalities (5) for surjective span and surjective semispan instead of span and semispan, respectively, do not hold. The example from [8], however, is not a tree-like continuum: it contains a simple closed curve. It is the aim of the present paper to describe an example of a tree-like continuum with the same span properties. Moreover, our example belongs topologically to the simplest, besides arcs, class of tree-like continua, namely, that of simple triods. By a simple triod we understand the union of three arcs having a common end-point and mutually disjoint except at that point.

Example. There exists a simple triod $X$ in the 3 -space such that $\sigma(X)=\sigma_{0}(X)=1$ and $\sigma^{*}(X)=\sigma_{0}^{*}(X)=\frac{1}{2}$.

Proof. The 3-space $R^{3}$ metrized by the ordinary Pythagorean distance will be used. Given two points $x, y \in R^{3}$, we denote by $\overline{x y}$ the straight-line segment with end-points $x$ and $y$. Setting

(6) $q_{i}=\left(\frac{1}{2} \cos \frac{2 \pi i}{3}, \frac{1}{2} \sin \frac{2 \pi i}{3}, 0\right), \quad r_{\imath}=\left(\cos \frac{2 \pi(i+1)}{3}, \sin \frac{2 \pi(i+1)}{3}, 0\right)$

$$
(i=1,2,3)
$$

and

$$
\begin{array}{ll}
s_{0}=\left(\frac{1}{4} \cos \frac{7 \pi}{12}, 0,0\right), & s_{1}=\left(\frac{1}{2} \cos \frac{7 \pi}{12},-\frac{1}{2} \sin \frac{7 \pi}{12}, 0\right), \\
s_{2}=\left(0,0, \frac{1}{4}\right), & s_{3}=\left(\frac{1}{2} \cos \frac{7 \pi}{12}, \frac{1}{2} \sin \frac{7 \pi}{12}, 0\right),
\end{array}
$$

we get ten points $q_{t}, r_{t}, s_{t} \in R^{3}$. Let $o=(0,0,0)$ be the origin. We take the polygonal arcs:

$$
\begin{aligned}
& S_{0}=\overline{o r_{2}} \cup \overline{r_{2} s_{2}} \cup \overline{s_{2} s_{0}} \cup \overline{s_{0} q_{1}} \cup \overline{q_{1} q_{2}}, \\
& S_{1}=\overline{o s_{1}} \cup \overline{s_{1} r_{1}}, \\
& S_{2}=\overline{o s_{3}} \cup \overline{s_{3} r_{3}},
\end{aligned}
$$

and we define $X$ to be the union

$$
X=S_{0} \cup S_{1} \cup S_{2}
$$


Clearly, $X$ is a simple triod in $R^{3}$ (Fig. 1).

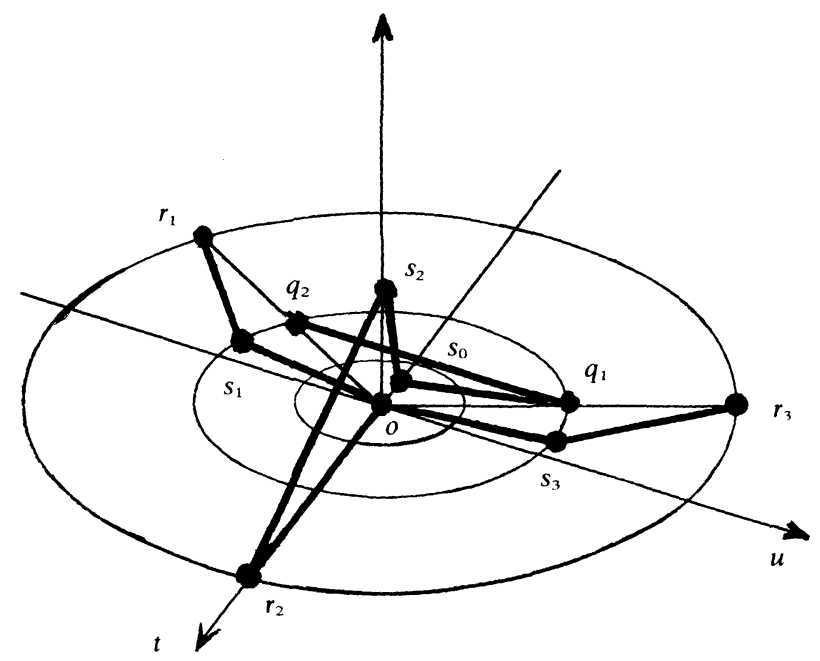

FIGURE 1

Let $A_{0}=\overline{o r_{2}}$ and $A_{i}=S_{l}$ for $i=1,2$. The set $T=A_{0} \cup A_{1} \cup A_{2}$ is also a simple triod. Its width (cf. [1] and [7]) is given by the formula ${ }^{1}$

$$
w(T)=\operatorname{Min}\left\{\operatorname{Max}\left\{\rho\left(x, A_{\imath+1} \cup A_{t+2}\right): x \in A_{\imath}\right\}: i=0,1,2\right\},
$$

where the subscripts of $A$, are taken mod 3 (see [7], Proposition 2.4).

Moreover, we have

$$
\operatorname{Max}\left\{\rho\left(x, A_{i+1} \cup A_{i+2}\right): x \in A_{\imath}\right\}=1 \quad(i=0,1,2),
$$

whence $w(T)=1$. It is known (see [9], p. 210) that $w(T) \leqq \sigma(T)$. Since $T \subset X$, we get $\sigma(T) \leqq \sigma(X)$, by (5). Thus $1 \leqq \sigma(X)$. We claim that $\sigma_{0}(X) \leqq 1$. If $\sigma_{0}(X)>1$, there would exist, by (2), a connected nonempty set $A \subset X$ such that $\sigma_{0}^{*}(A)>1$. Hence there would be a connected set $C_{1} \subset X \times X$ such that $1<\operatorname{dist}\left(x, x^{\prime}\right)$ for $\left(x, x^{\prime}\right) \in C_{1}$ and $p_{1}\left(C_{1}\right)=A$. By (6) and (7), each of the ten points $q_{t}, r_{t}$ and $s_{t}$ belongs to the sphere of radius 1 and center at the origin. Consequently, the set $X$ is contained in this sphere, too, and therefore $\operatorname{dist}\left(o, x^{\prime}\right) \leqq 1$ for $x^{\prime} \in X$. It follows that the origin $o$ cannot be a point of $p_{1}\left(C_{1}\right)$, whence $o \notin A$, i.e., $A \subset X \backslash\{o\}$. The point $o$ cuts the simple triod $X$ into the three components $S_{l} \mid\{o\}(i=0,1,2)$. Since $A$ is a connected set, there has to be a

${ }^{1}$ Here $\rho(x, A)$ denotes the greatest lower bound of distances between the point $x$ and all points of $\boldsymbol{A}$. 
subscript $j=0,1,2$ such that $A \subset S_{\jmath} \backslash\{o\}$. But then $A \subset S_{j}$ and $\sigma_{0}\left(S_{j}\right) \geqq$ $\sigma_{0}^{*}(A)>1$, by (2). This, however, is impossible because $S_{J}$ is an arc (see [8], Proposition 1.3). As a result, we obtain $\sigma_{0}(X) \leqq 1$, and $\sigma(X)=$ $\sigma_{0}(X)=1$, by $(3)$.

The width of the simple triod $X$, i.e.,

$$
w(X)=\operatorname{Min}\left\{\operatorname{Max}\left\{\rho\left(x, S_{t+1} \cup S_{t+2}\right): x \in S_{i}\right\}: i=0,1,2\right\}
$$

is less than the width of the simple triod $T$ contained in $X$. Indeed, it is not difficult to check (cf. Fig. 1) that

$$
\operatorname{Max}\left\{\rho\left(x, S_{i+1} \cup S_{i+2}\right): x \in S_{i}\right\}= \begin{cases}1 & i=0 \\ \frac{1}{2} & i=1 \\ \frac{1}{2} & i=2\end{cases}
$$

where, again, the subscripts are taken mod 3. Consequently, $w(X)=\frac{1}{2}$. We have $w(X) \leqq \sigma^{*}(X)$ (see [9], p. 210) . To complete the proof of all the properties of the example, it is now sufficient, by (4), to prove that $\sigma_{0}^{*}(X) \leqq \frac{1}{2}$.

Suppose, on the contrary, that $\sigma_{0}^{*}(X)>\frac{1}{2}$. Then there exist a number $\alpha_{0}>\frac{1}{2}$ and a connected set $C_{\alpha_{0}} \subset X \times X$ such that $\alpha_{0} \leqq \operatorname{dist}\left(x, x^{\prime}\right)$ for $\left(x, x^{\prime}\right) \in C_{\alpha_{0}}$ and $p_{1}\left(C_{\alpha_{0}}\right)=X$. The closure $C$ of $C_{\alpha_{0}}$ in $X \times X$ is a continuum, and we also have $\alpha_{0} \leqq \operatorname{dist}\left(x, x^{\prime}\right)$ for $\left(x, x^{\prime}\right) \in C$ and $p_{1}(C)=$ $X$. The existence of such a continuum $C$ will lead to a contradiction in each of a number of cases considered below. Before listing them, we need to establish some geometric properties of the polygonal arcs which form the simple triod $X$.

The arcs

$$
B_{0}=\overline{s_{0} q_{1}} \cup \overline{q_{1} q_{2}}, \quad B_{1}=\overline{s_{0} s_{2}} \cup \overline{s_{2} r_{2}},
$$

and $B=B_{0} \cup B_{1}$ are subarcs of the arc $S_{0}$. By (6) and (7), the points $q_{1}$, $q_{2}$ and $s_{0}$ belong to the sphere of radius $\frac{1}{2}$ and center at the origin. Thus

$$
\operatorname{dist}(o, x) \leqq \frac{1}{2} \quad\left(x \in B_{0}\right) .
$$

Let $f: X \rightarrow R^{3}$ be the projection of $X$ onto the $t$-axis (Fig. 1). By (7), any plane containing a point of the segment $\overline{s_{0} s_{2}}$ and perpendicular to the $t$-axis meets the simple triod $X$ in a set contained in the sphere of radius $\frac{1}{4}$ and center at the origin (Fig. 2). Consequently, the diameter of the set $f^{-1} f(x)$ does not exceed $\frac{1}{2}$ for $x \in \overline{s_{0} s_{2}}$. If $x \in \overline{s_{2} r_{2}}$, the point $f(x)$ is a point

${ }^{2}$ The lemma in [9] states that $w(X) \leqq \sigma(X)$, but its proof actually provides an estimate of the surjective span rather than the span. Hence $w(X) \leqq \sigma^{*}(X)$ for each simple triod $X$. 
of $\overline{o r_{2}}$ having a distance not greater than $\frac{1}{4}$ from $x$. No other point of $X$ projects onto $f(x)$ besides $x$ and $f(x)$ itself. Hence $f^{-1} f(x)=\{x, f(x)\}$ for $x \in \overline{s_{2} r_{2}}$, and we get

$$
\operatorname{diam} f^{-1} f(x) \leqq \frac{1}{2} \quad\left(x \in B_{1}\right) .
$$

Now, since the continuum $C$ is mapped onto $X$ by the projection $p_{1}$, the point $q_{2}$ belongs to $p_{1}(C)$. The set $p_{1}^{-1}(B) \cap C$ is a closed proper subset of $C$ which meets $p_{1}^{-1}\left(q_{2}\right)$. There exists a component $K$ of $p_{1}^{-1}(B) \cap C$ containing a point of $p_{1}^{-1}\left(q_{2}\right)$, so that $q_{2} \in p_{1}(K)$. We observe that the $\operatorname{arc} B$ has a degenerate boundary in $X$, namely $\left\{r_{2}\right\}$. The continuum $K$ meets the boundary of $p_{1}^{-1}(B) \cap C$ in $C$ (see [4], p. 172), whence $p_{1}(K)$ must contain a point of the boundary of $B$ in $X$, i.e., $r_{2} \in p_{1}(K)$. The continuum $p_{1}(K)$, however, is a subset of the $\operatorname{arc} B$ whose end-points are $q_{2}$ and $r_{2}$. Thus $B=p_{1}(K)$. We denote $g=$ $p_{1} \mid K$. Hence $g: K \rightarrow B$ is a continuous mapping of $K$ onto $B$.

The point $s_{0}$ is a common end-point of the $\operatorname{arcs} B_{0}$ and $B_{1}$ whose union is $B$. Let $\mathscr{K}_{i}$ denote the collection of all components of the set $g^{-1}\left(B_{i}\right)(i=0,1)$. Each component of $g^{-1}\left(B_{i}\right)$ meets the boundary of $g^{-1}\left(B_{i}\right)$ in $K$ (ibidem). Each point of the boundary of $g^{-1}\left(B_{\imath}\right)$ in $K$ is sent under $g$ into a point of the boundary of $B_{\imath}$ in $B$. The latter boundary is degenerate, namely it is $\left\{s_{0}\right\}$. It follows that

$$
g^{-1}\left(s_{0}\right) \cap Z \neq \varnothing \quad\left(Z \in \mathscr{K}_{1} ; i=0,1\right) .
$$

The sets $M_{l}(j=0,1)$, defined by the formulae:

$$
\begin{aligned}
& M_{0}=\left\{y \in g^{-1}\left(s_{0}\right): f p_{1}(y) \leqq f p_{2}(y)\right\}, \\
& M_{1}=\left\{y \in g^{-1}\left(s_{0}\right): f p_{1}(y) \geqq f p_{2}(y)\right\},
\end{aligned}
$$

are closed subsets of $K$ and their union is $g^{-1}\left(s_{0}\right)$. We denote by $\mathscr{K}_{i j}$ the subcollection of $\mathscr{K}_{t}$ consisting of all those elements of $\mathscr{K}_{i}$ which meet the set $M_{j}(i, j=0,1)$.

The remainder of the proof is divided into several cases, each of them ending with a contradiction. In this way, we shall prove that the surjective semispan of $X$ is, indeed, less than or equal to $\frac{1}{2}$.

Case 1. $\mathscr{K}_{00}=\varnothing$. Since $q_{2} \in B_{0} \subset B=g(K)$, the point-inverse $g^{-1}\left(q_{2}\right)$ is a nonempty subset of $g^{-1}\left(B_{0}\right)$. Let $K^{\prime}$ be a component of $g^{-1}\left(B_{0}\right)$ which meets $g^{-1}\left(q_{2}\right)$. Then $K^{\prime} \in \mathscr{K}_{0}$ and, by (10), the component $K^{\prime}$ also meets $g^{-1}\left(s_{0}\right)=M_{0} \cup M_{1}$. Hence it must meet $M_{0}$ or $M_{1}$, that is, $K^{\prime} \in \mathscr{K}_{00}$ or $K^{\prime} \in \mathscr{K}_{01}$, respectively. But $\mathscr{K}_{00}$ being empty, we obtain $K^{\prime} \in \mathscr{K}_{01}$. On the other hand, the continuum $g\left(K^{\prime}\right)$ is a subset of the arc 
$B_{0}$ whose end-points are $q_{2}$ and $s_{0}$. Since both $q_{2}$ and $s_{0}$ belong to $g\left(K^{\prime}\right)$, we have $B_{0}=g\left(K^{\prime}\right)$. Also $q_{1} \in B_{0}$, so that $q_{1} \in g\left(K^{\prime}\right)$. Let $y_{k} \in K^{\prime}$ be points such that $g\left(y_{k}\right)=q_{k}(k=1,2)$. It follows from the inclusions

$$
K^{\prime} \subset K \subset C \subset X \times X
$$

that $g\left(y_{k}\right)=p_{1}\left(y_{k}\right)$ and

$$
\frac{1}{2}<\alpha_{0} \leqq \operatorname{dist}\left[p_{1}\left(y_{k}\right), p_{2}\left(y_{k}\right)\right]=\operatorname{dist}\left[q_{k}, p_{2}\left(y_{k}\right)\right] \quad(k=1,2),
$$

where $p_{2}\left(y_{k}\right)$ is a point of the simple triod $X$. Observe that each point of the arc $S_{2}$ has a distance less than or equal to $\frac{1}{2}$ from $q_{1}$. Similarly, each point of the arc $S_{1}$ has a distance less than or equal to $\frac{1}{2}$ from $q_{2}$. Thus $p_{2}\left(y_{k}\right) \in S_{0} \cup S_{k}(k=1,2)$. As a result, the continuum $p_{2}\left(K^{\prime}\right)$ meets both sets $S_{0} \cup S_{1}$ and $S_{0} \cup S_{2}$ whose union $X$ contains $p_{2}\left(K^{\prime}\right)$. The continuum $p_{2}\left(K^{\prime}\right)$ must, therefore, intersect the common part of these two sets which is equal to $S_{0}$. We conclude Case 1 by proving that the conditions $K^{\prime} \in \mathscr{K}_{01}$ and $p_{2}\left(K^{\prime}\right) \cap S_{0} \neq \varnothing$ are incompatible. First, we note that $K^{\prime} \in \mathscr{K}_{01}$ means $K^{\prime} \cap M_{1} \neq \varnothing$, i.e., there is a point $y_{3} \in K^{\prime}$ with $g\left(y_{3}\right)=s_{0}$ and $f p_{2}\left(y_{3}\right) \leqq f p_{1}\left(y_{3}\right)$. Again, $g\left(y_{3}\right)=p_{1}\left(y_{3}\right)$. The $t$-coordinate of $p_{2}\left(y_{3}\right)$ is less than or equal to the $t$-coordinate of $s_{0}$; this is what the last inequality says. The point $p_{2}\left(y_{3}\right)$ is, of course, a point of $X$, and we see (cf. Fig. 1) that it can be located only on $B_{0}$ or $S_{1} \cup S_{2}$; the remaining points of $S_{0}$ have $t$-coordinates greater than the $t$-coordinate of $s_{0}$. However, we also have

$$
\frac{1}{2}<\alpha_{0} \leqq \operatorname{dist}\left[p_{1}\left(y_{3}\right), p_{2}\left(y_{3}\right)\right]=\operatorname{dist}\left[s_{0}, p_{2}\left(y_{3}\right)\right],
$$

and each point of the arc $B_{0}$ has a distance less than $\frac{1}{2}$ from $s_{0}$ (cf. Fig. 2). Hence $p_{2}\left(y_{3}\right) \in S_{1} \cup S_{2}$. If the continuum $p_{2}\left(K^{\prime}\right)$ intersected $S_{0}$, it would intersect both $S_{0}$ and $S_{1} \cup S_{2}$, so that it would have to intersect the common part of $S_{0}$ and $S_{1} \cup S_{2}$ which is the degenerate set $\{o\}$. In such a situation, there would exist a point $y_{0} \in K^{\prime}$ with $o=p_{2}\left(y_{0}\right)$, or, which is the same thing, a point $x_{0} \in X$ such that $\left(x_{0}, o\right) \in K^{\prime}$. Then $\frac{1}{2}<\alpha_{0} \leqq$ $\operatorname{dist}\left(x_{0}, o\right)$ and $x_{0} \in p_{1}\left(K^{\prime}\right)$. Since $p_{1}\left(K^{\prime}\right)=g\left(K^{\prime}\right) \subset B_{0}$, we would get $x_{0} \in B_{0}$, contradicting (8).

Case 2. $\mathscr{K}_{11}=\varnothing$. Since $r_{2} \in B_{1} \subset B=g(K)$, the point-inverse $g^{-1}\left(r_{2}\right)$ is a nonempty subset of $g^{-1}\left(B_{1}\right)$. Let $K^{\prime \prime}$ be a component of $g^{-1}\left(B_{1}\right)$ which meets $g^{-1}\left(r_{2}\right)$. Then $K^{\prime \prime} \in \mathscr{K}_{1}$, and $K^{\prime \prime}$ also meets $g^{-1}\left(s_{0}\right)=$ $M_{0} \cup M_{1}$, by (10). Hence $K^{\prime \prime} \in \mathscr{K}_{10}$ or $K^{\prime \prime} \in \mathscr{K}_{11}$. But $\mathscr{K}_{11}$ being empty, we obtain $K^{\prime \prime} \in \mathscr{K}_{10}$, which means that $K^{\prime \prime} \cap M_{0} \neq \varnothing$. Consequently, there is a point $z_{0} \in K^{\prime \prime}$ with $f p_{1}\left(z_{0}\right) \leqq f p_{2}\left(z_{0}\right)$. On the other hand, there exists a point $z_{2} \in K^{\prime \prime}$ such that $g\left(z_{2}\right)=r_{2}$. Thus $z_{2} \in K$ and $g\left(z_{2}\right)=p_{1}\left(z_{2}\right)$. The 
$t$-coordinate $f\left(r_{2}\right)$ of the point $r_{2}$ is equal to 1 , and it is the maximum $t$-coordinate of points belonging to $X$ (cf. Fig. 1). It follows that $f p_{2}\left(z_{2}\right) \leqq f\left(r_{2}\right)=f p_{1}\left(z_{2}\right)$. The continuum $K^{\prime \prime}$ then contains two points, $z_{0}$ and $z_{2}$, at which the real-valued functions $f p_{1}$ and $f p_{2}$ switch the order. As a result, there exists a point $z_{1} \in K^{\prime \prime}$ with $f p_{1}\left(z_{1}\right)=f p_{2}\left(z_{1}\right)$. We denote $x_{1}=p_{1}\left(z_{1}\right)$ and observe that $z_{1} \in K \subset C$. Therefore we have

$$
\frac{1}{2}<\alpha_{0} \leqq \operatorname{dist}\left[p_{1}\left(z_{1}\right), p_{2}\left(z_{1}\right)\right]=\operatorname{dist}\left[x_{1}, p_{2}\left(z_{1}\right)\right],
$$

where both points $x_{1}$ and $p_{2}\left(z_{1}\right)$ belong to the set $f^{-1} f\left(x_{1}\right)$. Then $\frac{1}{2}<\operatorname{diam} f^{-1} f\left(x_{1}\right)$. Since $p_{1}\left(K^{\prime \prime}\right)=g\left(K^{\prime \prime}\right) \subset B_{1}$, we get $x_{1} \in B_{1}$, which contradicts (9).

Case 3. $\mathscr{K}_{00} \neq \varnothing \neq \mathscr{K}_{11}$. By (10), we see that $\mathscr{K}_{i}=\mathscr{K}_{i 0} \cup \mathscr{K}_{i 1}$ $(i=0,1)$. Consequently, the continuum $K$ admits the decomposition ${ }^{3}$

$$
\begin{aligned}
K & =g^{-1}(B)=g^{-1}\left(B_{0} \cup B_{1}\right)=g^{-1}\left(B_{0}\right) \cup g^{-1}\left(B_{1}\right) \\
& =\left|\mathscr{K}_{0}\right| \cup\left|\mathscr{K}_{1}\right|=\left(\left|\mathscr{K}_{00}\right| \cup\left|\mathscr{K}_{10}\right|\right) \cup\left(\left|\mathscr{K}_{01}\right| \cup\left|\mathscr{K}_{11}\right|\right),
\end{aligned}
$$

where both sets $\left|\mathscr{K}_{00}\right| \cup\left|\mathscr{K}_{10}\right|$ and $\left|\mathscr{K}_{01}\right| \cup\left|\mathscr{K}_{11}\right|$ are nonempty. These two sets, however, are closed in $K$ (see [4], p. 182). Their common part must be nonempty, too, and Case 3 splits into four subcases.

Case 3(a). $\left|\mathscr{K}_{00}\right| \cap\left|\mathscr{K}_{01}\right| \neq \varnothing$. In this case, there exists an element $K^{*} \in \mathscr{K}_{00} \cap \mathscr{K}_{01}$. In particular, $K^{*} \in \mathscr{K}_{00}$ means $K^{*} \cap M_{0} \neq \varnothing$, i.e., there is a point $y^{*} \in K^{*}$ with $g\left(y^{*}\right)=s_{0}$ and $f p_{1}\left(y^{*}\right) \leqq f p_{2}\left(y^{*}\right)$. Thus $y^{*} \in K$ and $g\left(y^{*}\right)=p_{1}\left(y^{*}\right)$, whence $f\left(s_{0}\right) \leqq f p_{2}\left(y^{*}\right)$. The $t$-coordinate of $p_{2}\left(y^{*}\right)$ is, therefore, greater than or equal to the $t$-coordinate of $s_{0}$. The only points of the arcs $S_{1}$ and $S_{2}$ whose $t$-coordinates satisfy this inequality are those points of the segments $\overline{o S_{1}}$ and $\overline{o S_{3}}$, respectively, which belong to the sphere of radius $\frac{1}{4}$ and center at the origin (cf. Fig. 2). The point $s_{0}$ also belongs to this sphere, so that its distance to each of those points is less than or equal to $\frac{1}{2}$. On the other hand, it follows from $y^{*} \in K \subset C$ that

$$
\frac{1}{2}<\alpha_{0} \leqq \operatorname{dist}\left[p_{1}\left(y^{*}\right), p_{2}\left(y^{*}\right)\right]=\operatorname{dist}\left[s_{0}, p_{2}\left(y^{*}\right)\right]
$$

and $p_{2}\left(y^{*}\right) \in X$. We conclude that the point $p_{2}\left(y^{*}\right)$ cannot be located on $S_{1}$ or $S_{2}$. Hence $p_{2}\left(y^{*}\right) \in S_{0}$, and thus $p_{2}\left(K^{*}\right) \cap S_{0} \neq \varnothing$. Also, $K^{*} \in$ $\mathscr{K}_{01}$. It has been proved in Case 1 , for $K^{\prime}$ instead of $K^{*}$, that the last two conditions are incompatible.

\footnotetext{
${ }^{3}$ Given a collection $\mathscr{K}$ of sets, we denote by $|\mathscr{K}|$ the union of all elements of $\mathscr{K}$.
} 


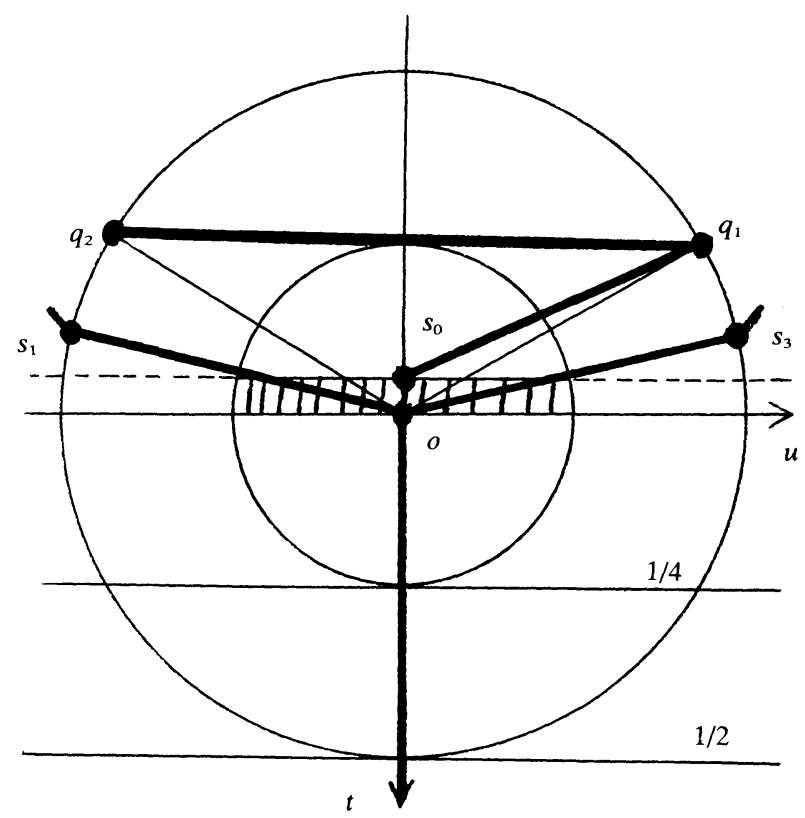

FIGURE 2

Case 3(b). $\left|\mathscr{K}_{10}\right| \cap\left|\mathscr{K}_{11}\right| \neq \varnothing$. In this case, there exists an element $K^{* *} \in \mathscr{K}_{10} \cap \mathscr{K}_{11}$. In particular, we have $K^{* *} \in \mathscr{K}_{1}$, whence $K^{* *} \subset K$ and $p_{1}\left(K^{* *}\right)=g\left(K^{* *}\right) \subset B_{1}$. Being an element of $\mathscr{K}_{1}$, the continuum $K^{* *}$ meets $M_{l}(j=0,1)$ and, by the definition of the sets $M_{l}$, it contains two points at which the functions $f p_{1}$ and $f p_{2}$ switch their order. We have shown in Case 2, for $K^{\prime \prime}$ in lieu of $K^{* *}$, that this combination of properties leads to a contradiction.

Case 3(c). $\quad\left|\mathscr{K}_{00}\right| \cap\left|\mathscr{K}_{11}\right| \neq \varnothing$. Let $K_{l} \in \mathscr{K}_{i i}(i=0,1)$ be elements that intersect, and let $c \in K_{0} \cap K_{1}$ be a point. Then $K_{i} \subset g^{-1}\left(B_{i}\right)$, whence

$$
g(c) \in g\left(K_{0}\right) \cap g\left(K_{1}\right) \subset B_{0} \cap B_{1}=\left\{s_{0}\right\}
$$

that is, $g(c)=s_{0}$. Thus $c \in g^{-1}\left(s_{0}\right)=M_{0} \cup M_{1}$, and $c \in M_{0}$ or $c \in M_{1}$. If $c \in M_{0}$, then $K_{1} \in \mathscr{K}_{10}$ and Case 3(c) reduces to Case 3(b). If $c \in M_{1}$, then $K_{0} \in \mathscr{K}_{01}$ and Case 3(c) reduces to Case 3(a).

Case 3(d). $\quad\left|\mathscr{K}_{10}\right| \cap\left|\mathscr{K}_{01}\right| \neq \varnothing$. Let $L_{0} \in \mathscr{K}_{01}$ and $L_{1} \in \mathscr{K}_{10}$ be elements that intersect, and let $d \in L_{0} \cap L_{1}$ be a point. As in Case 3(c), we obtain $d \in M_{0} \cup M_{1}$. If $d \in M_{0}$, then $L_{0} \in \mathscr{K}_{00}$ and Case 3(d) reduces to Case 3(a). If $d \in M_{1}$, then $L_{1} \in \mathscr{K}_{11}$ and Case 3(d) reduces to Case 3(b). 


\section{REFERENCES}

1. C. E. Burgess, Collections and sequences of continua in the plane II, Pacific J. Math., 11 (1961), 447-454.

2. W. T. Ingram, An atriodic tree-like continuum with positive span, Fund. Math., 77 (1972), 99-107.

3. - An uncountable collection of mutually exclusive planar atriodic tree-like continua with positive span, Fund. Math., 85 (1974), 73-78.

4. K. Kuratowski, Topology, vol. II, Academic Press 1968.

5. A. Lelek, Disjoint mappings and the span of spaces, Fund. Math., 55 (1964), 199-214.

6. - Sets of distances and mappings of certain continua, (to appear).

7. - The span and the width of continua, Fund. Math., (to appear).

8. - On the surjective span and semispan of connected metric spaces, Colloq. Math., (to appear).

9. A. Lelek and L. Mohler, Real-valued continuous functions and the span of continua, Colloq. Math., 32 (1975), 209-211.

10. D. Zaremba, On pseudo-open mappings, Proc. Third Prague Topological Symposium 1971, 469-470.

Received September 23, 1975.

WAYNE STATE UNIVERSITY 




\section{Pacific Journal of Mathematics}

Vol. 64, No. 1

May, 1976

Walter Allegretto, Nonoscillation theory of elliptic equations of order $2 n \ldots \ldots \quad 1$

Bruce Allem Anderson, Sequencings and starters.................. 17

Friedrich-Wilhelm Bauer, A shape theory with singular homology .......... 25

John Kelly Beem, Characterizing Finsler spaces which are

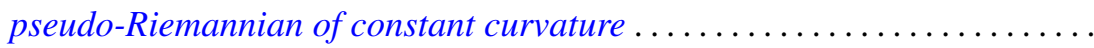

Dennis K. Burke and Ernest A. Michael, On certain point-countable

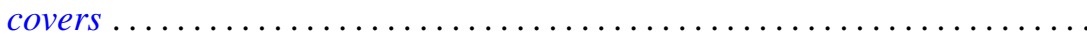

Robert Chen, A generalization of a theorem of Chacon ............... 93

Francis H. Clarke, On the inverse function theorem ................ 97

James Bryan Collier, The dual of a space with the Radon-Nikodým

property ....................................... 103

John E. Cruthirds, Infinite Galois theory for commutative rings ............ 107

Artatrana Dash, Joint essential spectra......................... 119

Robert M. DeVos, Subsequences and rearrangements of sequences in FK

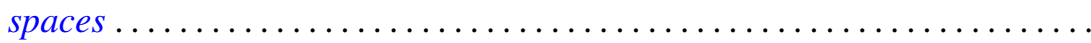

Geoffrey Fox and Pedro Morales, Non-Hausdorff multifunction generalization

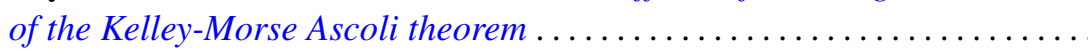

Richard Joseph Fleming, Jerome A. Goldstein and James E. Jamison, One

parameter groups of isometries on certain Banach spaces.............

Robert David Gulliver, II, Finiteness of the ramified set for branched

immersions of surfaces

Kenneth Hardy and István Juhász, Normality and the weak cb property ...... 167

C. A. Hayes, Derivation of the integrals of $L^{(q)}$-functions.

Frederic Timothy Howard, Roots of the Euler polynomials .

Robert Edward Jamison, II, Richard O'Brien and Peter Drummond Taylor, On

embedding a compact convex set into a locally convex topological vector space ....................................

Andrew Lelek, An example of a simple triod with surjective span smaller than span ...

Janet E. Mills, Certain congruences on orthodox semigroups

Donald J. Newman and A. R. Reddy, Rational approximation of $e^{-x}$ on the positive real axis.

John Robert Quine, Jr., Homotopies and intersection sequences ...

Nambury Sitarama Raju, Periodic Jacobi-Perron algorithms and fundamental units ....

Herbert Silverman, Convexity theorems for subclasses of univalent functions. . .

Charles Frederick Wells, Centralizers of transitive semigroup actions and endomorphisms of trees.........................

Volker Wrobel, Spectral approximation theorems in locally convex spaces ..................... 